Projets

de paysage

\section{Projets de paysage}

Revue scientifique sur la conception et l'aménagement de l'espace

13 | 2015

Biodiversité et paysage

\title{
Prise en compte de la diversité végétale arborée chez les paysagistes
}

Trois siècles d'histoire des jardins entre tradition et renouveau

A Description of Arborescent Plant Diversity among Landscape Architects Three Centuries of the History of Gardens between Tradition and Renewal

Brice Dacheux-Auzière, Audrey Marco et Yves Petit-Berghem

\section{OpenEdition \\ Journals}

Édition électronique

URL : http://journals.openedition.org/paysage/9411

DOl : 10.4000/paysage. 9411

ISSN : 1969-6124

\section{Éditeur :}

École nationale supérieure du paysage de Versailles-Marseille, Institut national des sciences appliquées Centre Val de Loire - École de la nature et du paysage, École nationale supérieure d'architecture et de paysage de Bordeaux, École nationale supérieure d'architecture et de paysage de Lille, Agrocampus Angers

Référence électronique

Brice Dacheux-Auzière, Audrey Marco et Yves Petit-Berghem, « Prise en compte de la diversité végétale arborée chez les paysagistes », Projets de paysage [En ligne], 13 | 2015, mis en ligne le 31 décembre 2015, consulté le 04 septembre 2020. URL : http://journals.openedition.org/paysage/9411 ; DOI : https://doi.org/10.4000/paysage.9411

Ce document a été généré automatiquement le 4 septembre 2020.

Projets de paysage 


\section{Prise en compte de la diversité végétale arborée chez les paysagistes}

Trois siècles d'histoire des jardins entre tradition et renouveau

A Description of Arborescent Plant Diversity among Landscape Architects Three Centuries of the History of Gardens between Tradition and Renewal

Brice Dacheux-Auzière, Audrey Marco et Yves Petit-Berghem

\section{NOTE DE L'AUTEUR}

Ce travail bibliographique et d'écriture est réalisé dans le cadre d'une thèse en science de l'environnement, articulant écologie et paysagisme. Elle est financée de 2015-2018 par la région Provence-Alpes-Côte d'Azur et s'intitule « Biodiversité et conception paysagère : le cas de la diversité végétale arborée et sa prise en compte dans la conception et la gestion des parcs urbains. Le cas de la ville de Marseille».

Depuis quelques années, les recherches en sciences de la vie et de l'environnement ainsi qu'en sciences humaines et sociales tendent à montrer l'importance et le rôle de l'arbre en ville dans la prise en compte des enjeux environnementaux. L'inscription de la conservation de la biodiversité dans les enjeux politiques de la fin du $\mathrm{xx}^{\mathrm{e}}$ siècle propose alors une nouvelle manière de considérer la nature en ville (Raymond et Simon, 2012). Aujourd'hui, cette dernière est représentée par les végétaux et notamment les arbres qui servent de support à une analyse des relations entre le citadin et la nature mais aussi de connectivité écologique entre les écosystèmes par l'intermédiaire des trames vertes (Clergeau, 2008 ; Arrif et al., 2011). Cet objet de nature, véritable support de communication pour la ville, considéré comme indispensable pour son cadre de vie, favorise la biodiversité et régule l'eau des sols (Arnould et al., 2011). Il permet à la diversité du vivant (et aux bénéfices qu'elle génère) d'être une solution opérationnelle face aux nouveaux défis (lutte contre l'îlot de chaleur urbain, captage 
CO2, filtration de l'air, cadre de vie, etc.) de la conception urbaine (Chiesura, 2004). La nature en ville en général et l'arbre en particulier sont donc à (re)penser (Arnould et al., 2011) en tenant compte de l'héritage des parcs et jardins des trois derniers siècles et ce qui les lie à l'acclimatation d'espèces exotiques (Clergeau, 2008) et aux explorateurs naturalistes. Ces derniers ont nourri la formidable explosion de l'inventaire du vivant au cours du XIX et du Xxe siècle (Arnould, 2006).

2 Ainsi l'héritage des grands domaines privés du XVII et $\mathrm{XVIII}^{\mathrm{e}}$ siècle, la tradition en matière d'urbanisme végétal qui accompagne le développement des villes à partir du XIX siècle (Mollie, 2009), et la création d'espaces à caractère de nature issus des politiques d'espaces verts puis des trames vertes au XXI siècle (Medhi et al., 2012) attribuent à la diversité arborée le statut de valeur ajoutée paysagère (Tollis, 2013). Cependant, que ce soit les grands axes plantés d'André Le Nôtre, ou les aménagements du parc des Buttes-Chaumont, dont les fonctions étaient politiques et hygiénistes, les éléments de nature présents dans les villes n'étaient pas considérés à l'époque pour ce qu'ils sont actuellement : des éléments du tissu vivant de la planète, des éléments et des vecteurs (ou supports) de biodiversité (Raymond et Simon, 2012). Pourtant ces éléments végétaux ont eu, et ont encore, des fonctions écologiques importantes pour les écosystèmes urbains. D'ailleurs, les espaces végétalisés urbains sont passés d'espaces monofonctionnels à vocation sociale ou urbanistique pendant la période de l'urbanisme fonctionnaliste à des espaces multifonctionnels mettant en exergue leur potentiel environnemental, social et urbanistique (Searns, 1995 ; Mehdi et al., 2012).

Si l'évolution des pratiques paysagistes et horticoles s'est manifestée par un glissement des formes ornementales vers des formes de nature spontanée (indigène ou naturalisée) (Aggeri et Donadieu, 2003), elle montre aussi l'écologisation des pratiques qui sous-tend la construction de nouveaux rapports à l'espace et à la nature (LégerSmith, 2014). La thèse d'Anaïs Léger-Smith soutenue en 2014 retrace à partir du XIX siècle la relation qu'il est possible d'apercevoir entre écologie et paysage et la manière dont les enjeux écologiques se sont progressivement intégrés dans le domaine de l'aménagement du paysage urbain. Par ailleurs, l'assemblage des espaces entre eux pour favoriser l'abondance et la biodiversité en ville (Arnould et al., 2011) est également un angle projectuel sur lequel le paysagiste intervient. Ces espaces sont porteurs d'une richesse biologique végétale au travers de laquelle l'arbre apporte une contribution, et non des moindres : il traverse les siècles et/ou les ères géologiques dans la diversité génétique, spécifique et écosystémique. La diversité des arbres dans l'espace, et ce à différentes échelles spatiales (locale, alpha; entre sites, beta ; régionale; gamma), est donc un point très important car le paysagiste, par le projet, manipule le végétal à toutes les échelles, du local au global ainsi que dans sa structuration et son fonctionnement. Le végétal est un terme trop général pour évoquer la possibilité que les écrits sur l'art des jardins nous indiquent une prise en compte antérieure d'un certain nombre de critères relevant d'une considération écologique chez les paysagistes avant le $\mathrm{XIX}^{\mathrm{e}}$ siècle. Cependant, l'arbre n'étant pas totalement absent des préoccupations des paysagistes à cette période, il est intéressant de se demander au travers des siècles précédents et depuis l'apparition du concept de biodiversité (milieu des années 1980) si nous pouvons déjà dégager des paramètres d'organisation, de structure et de fonctionnement qui témoignent avant l'heure d'une dimension de la diversité arborée, alors que celle-ci n'apparaîtra spécifiquement qu'après les années 1990. 
Sans avoir l'ambition de dresser un large panorama de l'histoire des pratiques paysagistes au regard de l'arbre, cet article vise à interroger la relation entre la diversité végétale arborée et la pratique du projet de paysage, spécifiquement sur les parcs et jardins (bosquets, alignements, groupes d'arbres, etc.). Cette interrogation sera conduite à travers la littérature existante d'histoire des jardins, publiée au cours de ces trois derniers siècles au regard de l'évolution des styles et des concepts émergents au cours du temps. Ces ouvrages ont tous eu à un moment donné un impact dans la réflexion et la pratique des paysagistes et notamment sur la manière dont ces derniers ont conduit l'arbre dans leurs projets d'aménagement. En ciblant précisément notre analyse sur les parcs et jardins et sur la manière dont le végétal y a été associé, celle-ci ne nous permet pas, à ce stade de la recherche, d'analyser les éléments d'ordre contextuel, liés à la dynamique des territoires. Ces derniers, extérieurs aux parcs et jardins, ont pu avoir une influence sur les manières de végétaliser ces espaces. Nous n'irons pas au-delà des années 1980 car il faudrait élaborer une autre méthode dépassant le cadre bibliographique pour traiter de cette grande question, de la relation entre la conception et la diversité arborée. Cela est lié notamment à l'évolution rapide du discours sur ce concept à la fois scientifique et popularisé (Le Guyader, 2008). Il s'agit en effet de la limite de l'exercice tout comme le fait que ce regard historique nécessiterait un travail plus fin pour mieux saisir les relations entre ce qui se passe dans les parcs et jardins et en dehors. La littérature que nous mobilisons dans cet article se réfère uniquement à des praticiens français qui ont structuré la pensée théorique et la manière de pratiquer les sciences du paysage (Donadieu, 2012), depuis le temps des jardiniers, puis des horticulteurs (jardinistes) jusqu'à celui des architectespaysagistes (Donadieu, 2009a). Bien qu'il soit possible de distinguer ces figures professionnelles paysagères à travers les siècles (Donadieu, 2009b), toutes ont un point commun : celui de transformer l'espace. Pour cette raison, nous utiliserons le terme de paysagiste, quelquefois précédé de celui de jardinier, tout au long de cet article sans véritable distinction. Ces trois temps retracent approximativement cette faste période des trois derniers siècles mettant en perspective quatre grandes «ères " ou courants qui ont non seulement fait évoluer des manières de penser et de dessiner les projets (Baridon, 1998), mais aussi de prendre en considération l'arbre dans la conception et la gestion de grands parcs et domaines privés du XvII siècle à ceux d'aujourd'hui, rendus publics et urbains (Mollie, 2009). Nous proposons donc d'observer la manière dont les concepteurs ont intégré et conduit l'arbre dans leurs aménagements selon les quatre moments que Michel Baridon (1998) développe dans son ouvrage :

- l'âge baroque, représenté par les travaux de Le Nôtre et de ses disciples, en se référant à l'ouvrage d'Antoine Joseph Dezallier d'Argenville (1709), un des seuls à la fin de cette période à avoir écrit et illustré l'art des jardins classiques ;

- l'ère de l'homme sensible, véhiculée par le marquis de Girardin et Jean-Jacques Rousseau (Girardin, 1777);

- l'ère industrielle illustrée par Adolphe Alphand dans les promenades de Paris (Alphand, 1867-1973), puis Jean-Pierre Barillet-Deschamps, son disciple (Limido, 2002) et Édouard André (André, 1879) ;

- enfin notre ère, celle de la période d'avant-guerre avec Jean Claude Nicolas Forestier (Forestier, 1908) et celle de la politique des espaces verts d'après-guerre positionnant des paysagistes qui se sont illustrés dans la période du Mouvement moderne. C'est aussi le moment des grandes prises de conscience écologiques après les années 1970 où se 
distinguent les paysagistes Gilles Clément et Allain Provost dans la création du parc AndréCitroën.

5 À travers ce regard historique nous nous intéressons plus précisément au lien entre la diversité arborée et la conception d'un point de vue esthétique et géotechnique. Cela renvoie à se poser les questions suivantes: De quelles manières les arbres étaient-ils associés entre eux? Quelles ambiances cela produisait-il? Quels étaient les connaissances des sols et les moyens techniques mis en œuvre pour le développement des arbres? Nous nous intéressons également à la relation entre la diversité arborée et la reconstitution des milieux écologiques (dimension fonctionnelle de l'écologie) car la réalisation d'un jardin ou d'un parc est un artifice. Cela fait référence aux questions suivantes : Y avait-il une attention particulière à la diversité biologique des milieux reconstitués? Dans quelles mesures les espèces arborées étaient-elles pensées en termes d'interaction dans leur environnement « artificiel »?

6 Nous pensons dans un premier temps que les multiples traités de l'art des jardins au cours des siècles ont toujours eu une attention particulière à développer un chapitre relevant de l'arbre et des boisements. Nous supposons dans un second temps que malgré la pression sur le traitement de l'espace pendant la période de l'urbanisme fonctionnaliste, le paysagiste a su garder un regard sur l'art de connaître les arbres, mais écarte un certain nombre de paramètres liés à l'évolution de la société industrielle. Dans un troisième et dernier temps, nous présumons que les concepts émergents au cours des trente dernières années tendent à réinterpréter d'une manière contemporaine le bon sens des traités de l'art des jardins au regard de la prise en compte de l'arbre, de son environnement et des erreurs du passé.

\section{L'ère baroque (XVII' siècle) : l'arbre forestier, la ligne et la constitution d'une forêt}

7 L'âge baroque, marqué par le classicisme et les grands travaux de Le Nôtre à Versailles, donne le ton d'un courant de pensée dans lequel l'art surpasse la nature en l'esthétisant (Brunon, 2001). La régularité déployée dans les jardins témoigne des signes de grandeur et de noblesse recherchés à cette époque. Jusqu'au $\mathrm{Xv}^{\mathrm{e}}$ siècle, les sciences naturelles étaient encore très inspirées des essais de Pline l'Ancien et Columelle (naturaliste et agronome romain $\mathrm{du} \mathrm{I}^{\mathrm{er}}$ siècle après $\mathrm{J} .-\mathrm{C}$ ) ainsi que celui de Théophraste (philosophe, botaniste et naturaliste grec du II siècle avant J.-C) (Quellier, 2005). La connaissance du végétal commençait à s'affirmer et à s'incarner dans la pratique de l'art des jardins pour ainsi mieux comprendre le fonctionnement des végétaux. Les recherches de Henri Louis Duhamel du Monceau (botaniste et agronome), mais aussi celles d'Antoine de Jussieu (botaniste) au début $\mathrm{du} \mathrm{XVIII}^{\mathrm{e}}$ siècle, inspirées des travaux passés du père fondateur de la botanique, Joseph Pitton de Tournefort (Allorge, 2003), peuvent en témoigner ainsi que les travaux des botanistes Nehemiah Grew (1682, anglo-saxons) et Marcello Malpighi (1676, italien) qui jettent les bases de l'organographie végétale ${ }^{1}$. 


\section{L'arbre dans sa dimension forestière}

8 Dans la troisième partie de l'ouvrage La Théorie et la pratique du jardinage, Antoine Dézallier d'Argenville (naturaliste, historien d'art parfois considéré comme l'héritier de Le Nôtre) distingue deux sortes de groupements d'arbres :

- «les sauvages », appelés ainsi car ce sont ceux retrouvés couramment dans les bois et les forêts, comme le chêne, l'orme, le châtaignier, le charme, le tilleul, etc.;

- «les aquatiques», nommés de cette sorte car ils se plaisent davantage dans les sols gorgés d'eau tels que les trembles, le peuplier blanc, l'aulne, etc.

Cette distinction permet d'identifier deux milieux ou plus globalement deux environnements (forestier et marécageux) dans lesquels se distinguent des groupements végétaux arborés que les jardiniers-paysagistes du $\mathrm{xVII}^{\mathrm{e}}$ siècle ont réimplantés dans les jardins selon les règles de composition et le style de l'époque (Mosser et Teyssot, 2002).

10 Les observations naturalistes des différentes espèces d'arbres dans leur milieu ont abouti à une connaissance fine de la morphologie, des exigences, des usages et de l'utilisation des arbres. Ainsi, le chêne « est, pour ainsi dire le roi des Arbres [...] il jette un pivot en terre [...] il est plus propre à former dans les forêts et dans les bois, qu'à former des allées bien droites [...] son fruit se sème ou se pique en terre; c'est par là qu'il se perpétue » (Dezallier d'Argenville, 1709, p. 163).

11 Le choix des essences d'arbres à planter dans les jardins semble reposer sur de nombreux critères tels que l'assemblage et la composition souhaitée, les besoins de prospective racinaire ainsi que la situation et la qualité du terrain (humide/sec). Par conséquent, "il faut examiner par des fouilles faites en plusieurs endroits, le fond naturel de la terre, et regarder les herbes qui la couvrent. Si la terre se trouve humide, et qu'elle est couverte de roseaux, on y plantera des arbres aquatiques, si elle est sèche, les arbres appelés sauvages » (ibid., p. 190). Cette technique basée sur des observations des plantes indicatrices de terrain transparaît déjà dès le début $d u$ XVII ${ }^{\mathrm{e}}$ siècle dans l'ouvrage d'Olivier de Serres (agronome français, 1539-1619) intitulé Le Théâtre d'agriculture et mésnage des champs. Il dit alors que les « bons \& menus herbages croiffans naturellement és champs, vous aideront beaucoup à ceci car jamais bonnes et franches herbes, que les bestes mangent avec apetit, ne viennét abondamment és terres de peu de valeur [...] » (Olivier de Serres, 1600, p. 5).

\section{Le savoir forestier au service du projet}

Dans la troisième partie de l'ouvrage de Dezallier d'Argenville, nous dénombrons plusieurs sortes de compositions arborées dans les jardins de cette époque (notamment celui du jardin du roi à Versailles comme ci-dessous) et une diversité de manières de les mettre en œuvre :

- les forêts, les grands bois de haute futaie, les bois, les quinconces, les bois «verds" (persistants) et les bois taillis, se sèment ordinairement sur un sol labouré de différentes graines et fruits ou à partir de jeunes plants enracinés. L'espacement entre les plants varie en fonction de l'effet recherché (futaie, port libre ou taillis, buissonnant) ;

- les bosquets de moyenne futaie à haute palissade et les bosquets découverts ou à compartiment sont plantés en lisière d'arbres suffisamment hauts pour les conduire en palissade tandis que le cœur est garni de gazon (boulingrin); 
Figure 1. Vue de l'Arc de triomphe dans un des bosquets des jardins de Versailles

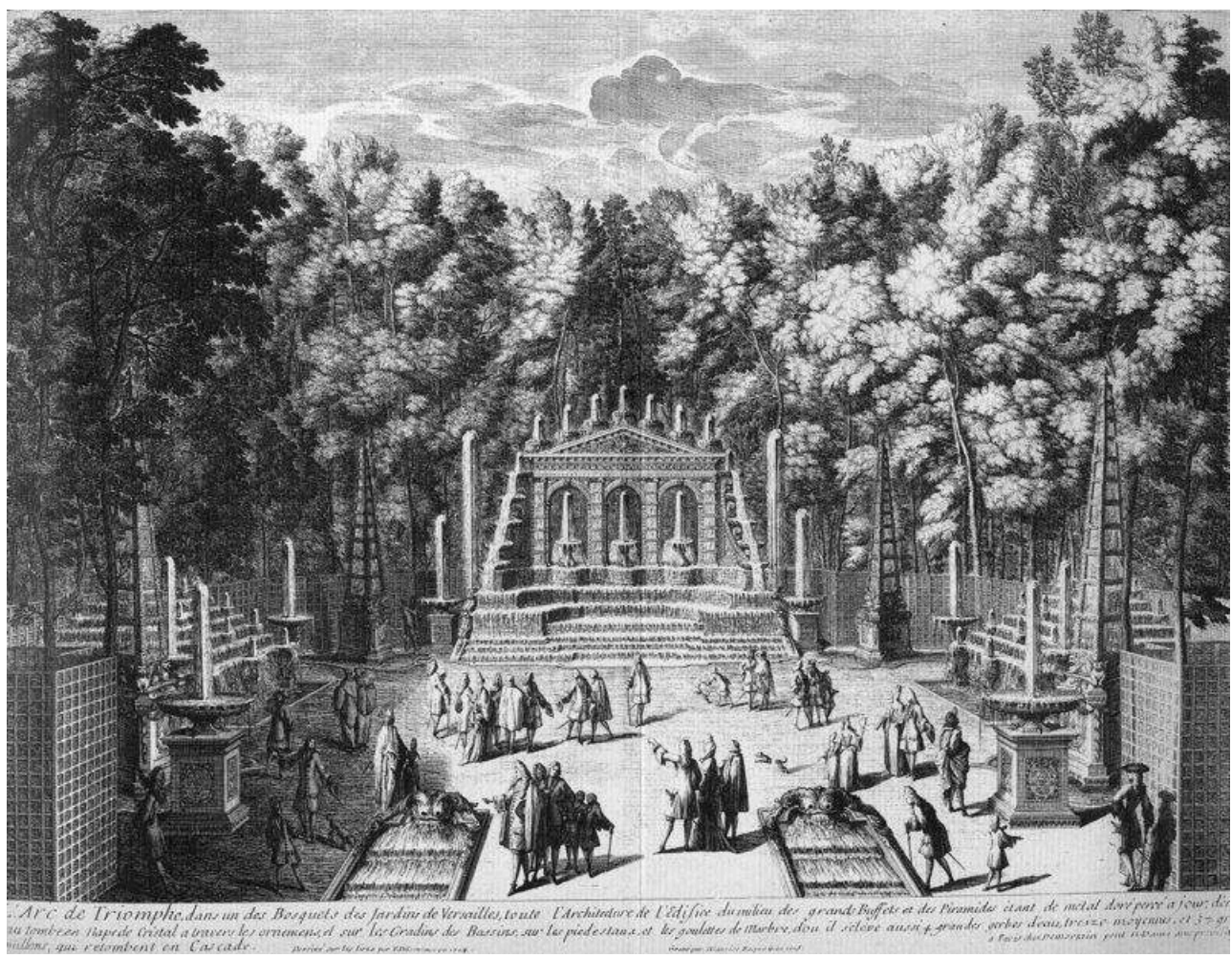

Source : Dezallier d'Argenville, 1709.

- les allées constituées d'arbres plus ou moins hauts, formés en palissade ou en haute futaie, sont taillées de part et d'autre et issues de plantations élevées en pépinière jusqu'à atteindre une circonférence à un mètre du collet comprise entre 14 et $16 \mathrm{~cm}$.

Du reste, le naturaliste Duhamel de Monceau trouve qu'il s'agit «d'une fort bonne pratique que de répandre beaucoup de fruits d'aubépine, puis d'azéroliers et de buissons-ardents dans les semis des bois car les arbrisseaux qui ne font aucun tort au chêne ni au châtaignier, couvrent la terre, font périr l'herbe [...]" (Duhamel de Monceau, 1755, p. 18).

Nous pouvons par conséquent objectiver un certain nombre d'observations. Visiblement, la tendance à cette époque d'esthétiser la nature par le jardin a créé des générations de jardiniers-paysagistes soucieux des observations de terrain menées en dehors des jardins, dans les forêts royales et au courant des recherches liées à la nature. Clemens Alexander Wimmer (historien des jardins allemands) évoque d'ailleurs l'inspiration suscitée par la variété et la symétrie qu'il est possible d'apercevoir dans les arbres (1989). Le jardin à la française rivalise alors de symétries et de variations avec la nature mais peut aussi être décrit conformément à la théorie des signatures ${ }^{2}$ (Oesterle, 1997). Les associations d'arbres se sont faites selon les caractéristiques de chaque individu (mode de croissance, type de multiplication, longévité, etc.), les formes souhaitées (boisements, alignements, formes géométriques variées...), leurs exigences et/ou leur rôle écologique (besoin d'humidité/assécher des espaces...) et les effets recherchés (ombrage, ouvert sur le ciel, fermé) afin de répondre au mieux à la composition générale du plan. 

d'inspiration forestière (perspective, choix des arbres, etc.) (Buridant, 2005) et l'attention particulière aux savoirs forestiers ont permis, sans que les jardinierspaysagistes ne s'en rendent compte, la création d'un milieu tout à fait artificiel, très proche d'une phytocénose forestière à l'écart des usages traditionnels (pacage, fagotage, coupe...). Ces derniers pratiqués essentiellement par les artisans et les paysans touchaient parfois les grands domaines (exemple: Versailles) car le braconnage, le vol, et les détériorations sont fréquents à cette époque (Quenet, 2015). Ce terme de phytocénose n'apparaîtra que dans les années 1990 définissant l'ensemble d'une communauté végétale vivant dans un espace écologique donné (le plus souvent un biotope).

17 Il semble que le temps accordé à l'arbre (depuis sa germination) et les soins apportés avant, pendant et après la plantation résument le dessein de cette faste période, celui de traverser les siècles.

\section{L'âge de l'homme sensible (XvIII siècle) : l'arbre sauvage, la courbe et la reproduction de la nature}

18 L'âge de l'homme sensible se manifeste par l'idée de retrouver une nature sylvestre dans les jardins, proche d'un idéal arcadien. Ce paradigme dérive au cours des décennies (selon le degré de présence de l'art) en suivant les courants littéraires (Gilpin, Rousseau, Burke, Pope, etc.) et l'évolution des sciences naturalistes marquées par Georges-Louis Buffon (naturaliste français), Carl von Linné (naturaliste suédois), puis plus tard Jean-Baptiste de Lamarck (botaniste français) et Duhamel du Monceau. Ce dernier demeure toujours une référence à cette époque. Cette période est également celle des grandes expéditions botaniques (Cook, Bougainville, frères Jussieu...) et de l'expansion des jardins des plantes, accueillant des espèces venues des quatre coins $\mathrm{du}$ monde (Allorge, 2003).

\section{L'arbre dans sa dimension pittoresque}

19 Comme au siècle passé avec Dezallier d'Argenville, Girardin (créateur des jardins d'Ermenonville, paysagiste) énonce dans son ouvrage une typologie des groupements d'arbres en distinguant ces derniers de trois sortes:

- les arbres forestiers, équivalant aux arbres sauvages (mêmes essences), destinés à produire de grandes masses boisées ;

- les arbres aquatiques (idem) ; 
- les arbres montagnards tels que les bouleaux, les pins et les cèdres. Cette dénomination qui n'était pas présente dans les jardins classiques est certainement liée à l'influence des peintres du sublime, cherchant à saisir les splendeurs immenses et inaccessibles de la nature.

Malgré une légère évolution de la typologie des groupements d'arbres, il n'y a pas de réel changement dans la palette utilisée par les paysagistes. Par ailleurs, l'engouement pour les plantes exotiques (Rozier et Mongez, 1779; Duchesne, 1761, dans la base de données Hortus ${ }^{3}$ ) reste assez controversé car « les arbres étrangers, sont non seulement très difficiles et très chers à élever, encore plus difficiles à conserver, et se lient toujours mal aux arbres du pays » (Girardin, 1777 et 2014, p. 73).

Cependant, comme le décrit Girardin :

« [... ] le coloris seul vous fera bien sentir l'effet de la perspective, la disposition des différents plans, [...] et vous indiquera le choix des arbres convenables à l'effet des masses principales de vos plantations (sous-entendu masse d'arbres forestiers)» (ibid., p. 28).

«Pour ordonnancer ce vaste tableau sur le terrain» (ibid., 1777), le paysagiste s'attache généralement à disposer les grandes masses des arbres forestiers au premier plan pour ménager des effets de perspective car «les bois sont la plus noble parure de la terre, leurs ombrages en font l'asile le plus naturel et le plus agréable ( (ibid., p. 71).

Figure 2. Hubert Robert (peintre et paysagiste), Le château et le parc de Méréville (Essonne) (réalisée entre 1784 et 1793)

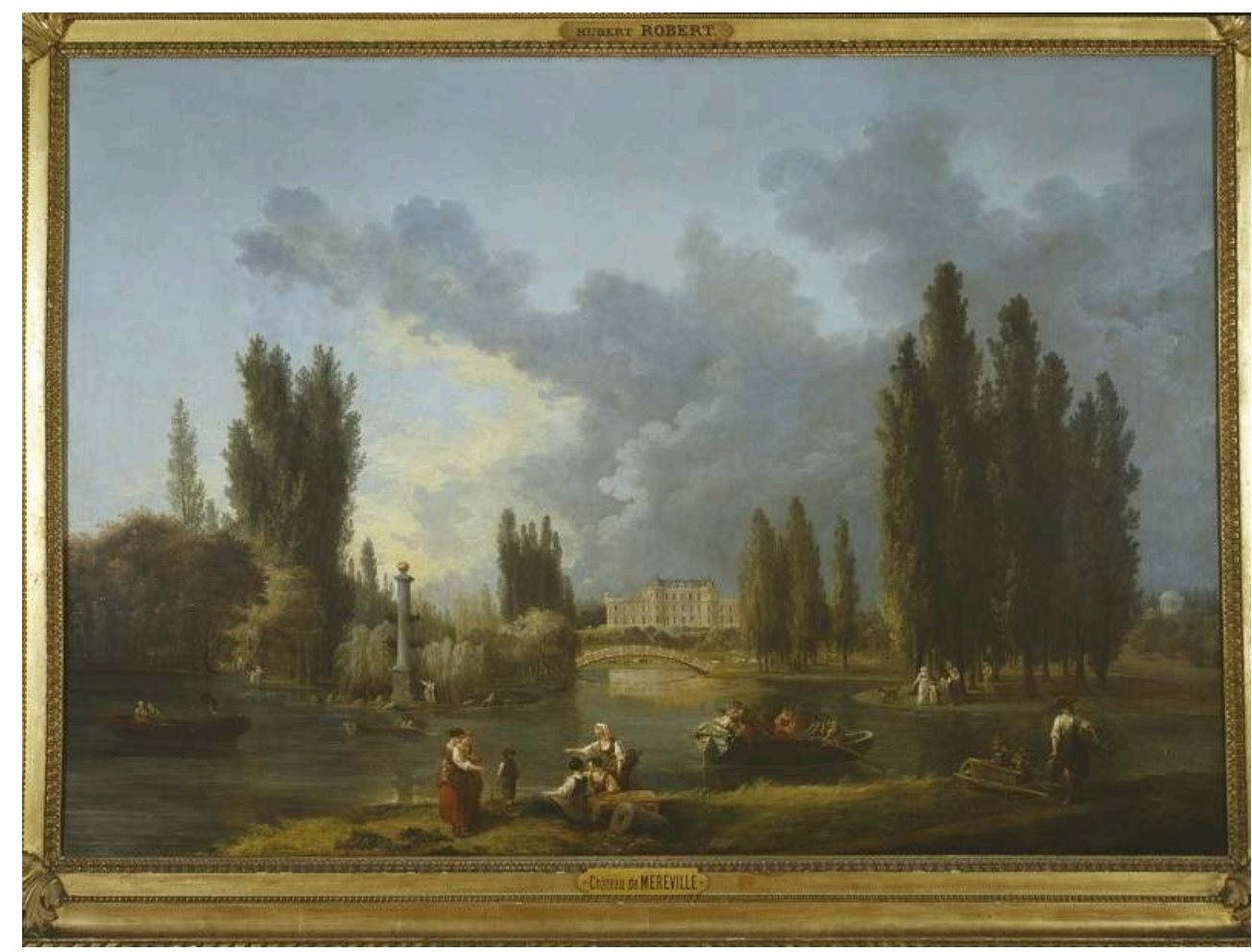

Source : Musée du domaine départemental de Sceaux. 


\section{L'arbre au cœur du dispositif d'imitation} " paysager $n$. Cet art des jardins se développe sous l'impulsion des grands travaux " pardins se développe sous l'impulsion des grands travaux entrepris en Europe sous Napoléon III (Paris) et en Amérique (New York) par les réflexions de Frederick Law Olmsted (architecte-paysagiste américain). En France, le courant de pensée vise à reproduire une nature maitrisée par l'homme où chaque 
élément est soumis à sa volonté et à sa puissance à l'image de la grande nature humanisée (Hautecœur, 1959). Georges Eugène Haussmann, chargé de l'application du dessein impérial, confie à l'ingénieur Alphand le soin de mettre en place un important service des Promenades et y installe le paysagiste Barillet Deschamps (Mollie, 2009).

Les parcs sont alors conçus pour mettre en scène le spectacle de la campagne environnante, les vues sont ménagées, les arbres forment des « coulisses " pour mettre en valeur les premiers plans et reculer les derniers (André, 1879). La connaissance botanique atteint son apogée, les paysagistes s'intéressent à la répartition des essences parmi plus de 90000 espèces connues (contre 10000 sous Linné). La théorie de l'évolution de Lamarck et celle de Charles Darwin ont eu une portée indiscutable dans la société et le domaine des sciences naturelles.

Par ailleurs, les voyages d'Alexander von Humboldt (naturaliste et explorateur allemand) et son Essai sur la géographie des plantes (1805) amorcent une science du paysage, par-delà les oppositions nature/culture, pays/paysage, temps/espace, science/esthétique/art (Brunon et al., 2009). De cet essai à celui de la Géographie botanique raisonnée d'Alphonse de Candolle (botaniste Suisse), une science nouvelle s'est constituée en établissant solidement les lois scientifiques de distribution des végétaux à la surface de la terre (Deleage, 1992).

\section{L'emploi systématique de l'arbre exotique}

L'évolution des connaissances en botanique (et en connaissance des milieux), en agronomie (nature et fonctionnement des sols) et le progrès technologique (les serres, les chaufferies, les caisses mobiles de transport des arbres, l'instrument Mazure ${ }^{4}$ pour analyser les terres) ont permis de dresser les listes de plantes (arbres, arbustes, espèces vivaces, etc.) selon plusieurs critères : la nature des sols, le climat, la rusticité des espèces, l'aspect décoratif (floraison, feuillage, comestible) et les usages (promenades plantées, parcs). La mode à cette époque, dans les parcs, est à l'exotisme. Aussi, «la nature végétale n'a plus de secret pour l'horticulteur » lit-on dans la Revue Horticole (Sahut, 1859, p. 88). Désormais séparée de l'agriculture, l'horticulture trouve dans les jardins un espace permanent d'exposition (Limido, 2002). Elle a ainsi industrialisé sa production pour réduire ses coûts et fournir principalement la demande en espèces exotiques. Les végétaux (surtout les arbres) sont alors exposés, associés entre eux pour séduire, étonner, avec l'idée principale de faire voyager le public dans des pays et des milieux naturels du monde entier.

À cette époque, le choix systématique d'essences exotiques par les paysagistes fait souvent l'objet de critiques comme celles de l'ingénieur en chef des Promenades, déplorant l'emploi systématique de ces essences (ibid., 2002). Barillet-Deschamps, alors premier jardinier en chef du service des Promenades et Plantations de la ville de Paris lui répond, dans une lettre : «[...] notre flore est très pauvre en végétaux d'ornements indigènes à la France, [...] la plupart de ceux que nous cultivons passent pour aborigènes parce qu'ils sont introduits depuis longtemps [...]/ » (Limido, 2002, p. 134.)

\section{L'arbre au service de la ville et de ses maux}

Comme l'évoque Luisa Limido tout au long de son ouvrage consacré à L'Art des jardins sous le Second Empire, les grands arbres dans les massifs, en lisière de parc et le long des 
allées (essences exotiques dominantes) sont plantés au centre et transportés par les chariots. Ils sont accompagnés d'arbres de 10 à $20 \mathrm{~cm}$ de diamètre (gros sujet) puis d'un gradient d'arbustes destinés à augmenter le volume apparent. Lorsqu'il s'agit de laisser une transparence entre les espaces, le pied des arbres est engazonné ou planté d'une plate-bande exotique.

Figure 3. A.-V. Deroy, Vue à vol d'oiseau du parc des Buttes-Chaumont, 1860

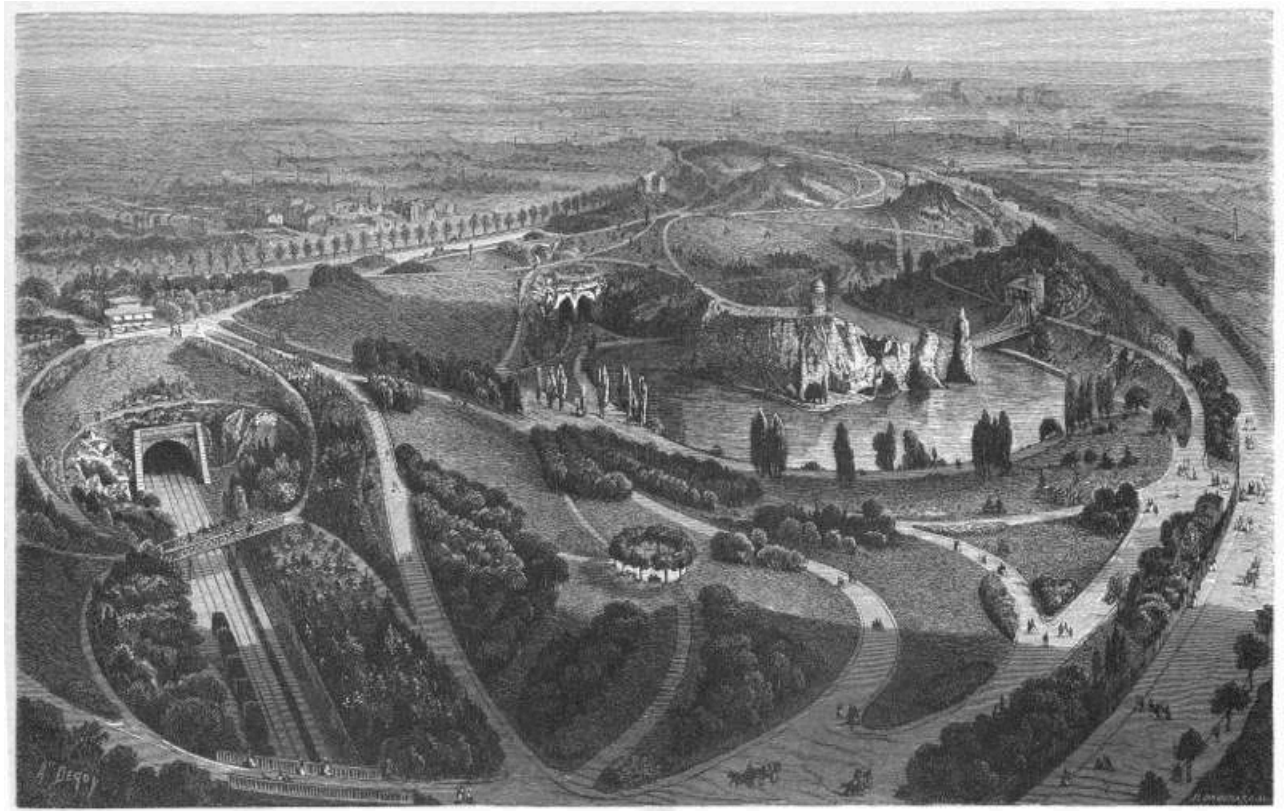

Source : Alphand, 1867. espèces revient à l'architecte-paysagiste : «Cette tâche réclame une mémoire sûre, du jugement et de l'expérience. J'ai fait remarquer que ces trois conditions étaient rarement remplies, et que la majeure partie des plantations dans les jardins était exécutée avec la plus grande indifférence. Il n'est pas rare de trouver un architectepaysagiste préparant seulement les listes de plantations d'après le catalogue d'un pépiniériste voisin, et ne se préoccupant guère, par des combinaisons dans les hauteurs, les formes et les couleurs des végétaux, des effets futurs de son travail.» (André, 1879, p. 581.)

richesse en espèces végétales dans les parcs et jardins, voire même dans tous les territoires français et européens puisque ce modèle s'est diffusé en province. Mais, force est de constater que peu d'attention est portée par les paysagistes à l'association des espèces entre elles et à leurs interactions comme cela a pu être le cas lorsqu'ils s'intéressaient davantage aux savoirs forestiers. L'arbre est alors la pièce maîtresse du décor urbain et une des solutions à ce qu'énonçait la politique hygiéniste : un nouveau

Projets de paysage, 13 | 2015 
cadre de ville mis en place par un urbanisme végétal (Mollie, 2009), ce que va prolonger le Mouvement moderne.

\section{L'ère moderne ( $x x^{\mathrm{e}}$ siècle) : l'arbre « vert », le vide et la masse boisée}

$\mathrm{Au}$ début $\mathrm{du} \mathrm{xx}^{\mathrm{e}}$ siècle, les paysages et les territoires sont en pleine mutation, les villes ne cessent de croître, grignotant les périphéries aux dépens des terres agricoles. Le mouvement réformateur dans les domaines de l'architecture et des arts décoratifs (Arts and Crafts 1880-1910) en Angleterre positionne quelques figures du paysagisme moderne telles que William Robinson. Culturaliste, ami de Frederick Law Olmsted et d'Édouard André, il réintroduit la culture de la flore indigène et préconise son association à des espèces exotiques dans les jardins. Son ouvrage intitulé The Wild Garden fait aujourd'hui écho aux valeurs environnementales de la ville durable (Aggeri, 2004). Par ailleurs, le mouvement des arts décoratifs du début du $\mathrm{XX}^{\mathrm{e}}$ siècle (1910-1930) en France propose le retour à la rigueur classique que de nombreux paysagistes vont suivre (Jean Claude Nicolas Forestier, André et Paul Véra, Achille Duchêne, etc.). Ainsi, Forestier (architecte-paysagiste français) développe une théorie selon laquelle la ville doit se doter d'un système de parcs distribué efficacement et de manière uniforme pour les besoins des citadins (Forestier, 1908). Lui-même, puis Jacques Gréber (architecte français, créateur de jardins), engagés dans les problématiques paysagères, héritiers d'un urbanisme soucieux d'articulation historique et géographique (culturaliste) s'opposent à leurs détracteurs (progressistes) fonctionnalistes (Blanchon, 2007) représentés par Le Corbusier et son discours fondé sur «le progrès, l'hygiène et la modernité ».

Par ailleurs, à partir de 1925, naît l'écologie urbaine, date à laquelle un recueil d'articles intitulé The City de l'école de Chicago est publié, invitant à étudier la ville en tant qu'elle est le produit de la nature. Du côté des écologues, nous constatons que se développe, dans les années 1960, une approche écosystémique de la ville intégrant le rapport ville/nature (Blanc, 1998). Enfin, dans les années 1980, les praticiens paysagistes renouvellent un certain nombre de fondements et reconnaissent le rôle du paysage minéral et végétal dans l'intervention urbaine mais aussi l'intérêt des infrastructures dans l'esthétique et la fonction des villes (Pousin, 2005).

\section{De l'arbre au cœur d'un système à l'arbre dans l'espace ouvert}

Le système de parc de Forestier, décliné en huit types d'espaces, énumère en réalité un gradient de nature, allant de l'espace naturel protégé ou exploité (pâturage, bois forestier...), composé de grands arbres forestiers encore très peu touchés par les activités humaines; puis à l'espace de nature périphérique proche, composé d'un patrimoine végétal arboré riche à l'image des jardins de Versailles; et enfin, à l'espace intra-urbain où la nature y a été mise en scène selon les époques mais où l'arbre a toujours été une priorité pour inscrire les projets dans le temps.

Les avenues promenades pour les uns, les parkways (avenue résidentielle peuplée d'arbres) pour les autres mettent par conséquent en réseau tous ces espaces entre eux par un système de contre-allées engazonnées plantées d'arbres. Ce système est 
toutefois décliné par Frederick Law Olmsted en Amérique à la fin du XIX siècle, après avoir observé l'avenue de l'Impératrice en France (Werquin et Demangeon, 1997) mais se diffuse plus largement dans les villes au début du $\mathrm{xx}^{\mathrm{e}}$ siècle.

Cependant, la période des Trente Glorieuses et de l'urbanisme fonctionnel donnent le ton d'une nouvelle manière de penser l'espace que les jeunes diplômés (section du paysage et de l'art des jardins créée au lendemain de la Seconde Guerre mondiale) de l'École d'horticulture de Versailles vont réinterpréter. Jean Bossu, architecte du Mouvement moderne, témoigne sur la façon de percevoir l'espace ouvert, fondée sur l'idéologie de Le Corbusier: "La nature s'étale en vainqueur sur ses plans (Le Corbusier) ; mieux que cela, ses villes assiègent la nature. [...] Il fallait un thème simple, scolaire. Sa présence suffisait : jardins à l'anglaise strictement, pelouses, fleurs, arbres [...] (Bossu et al., 1966, p. 14)

42 Cette forme simplifiée de la nature renvoie à une vision immatérielle et idéologique qui conduira à la politique des espaces verts menée jusqu'à la fin des années 1980 . Cependant, l'examen effectué, par Bernadette Blanchon, des réalisations paysagistes de l'après-guerre permet d'identifier trois étapes étalées de 1945 à 1975. Dans les années 1950, les paysagistes-planteurs, selon l'expression de Françoise Dubost, devaient l'essentiel de leurs inspirations à la bible de la profession qu'était l'ouvrage d'Édouard André écrit en 1878 (Donadieu, 1996).

Par-delà la fonction de "mettre du vert » dans les «blancs » du plan-masse (Dubost, 1994), les paysagistes ont transposé l'idée de percevoir le végétal comme un régulateur de ville (Donadieu, 1996), terme encore rattaché à l'hygiénisme du XIX siècle, à un végétal comme médiateur social, pour ainsi affirmer l'identité et la qualité de la ville. Ce n'est que par ce moyen que l'agglomération devient cité appropriée ou appropriable par ses habitants (ibid.).

\section{L'arbre support d'un génie écologique}

Par ailleurs, les villes nouvelles de la Grande Motte (Balladur et al., 1989) (début des travaux en 1965), et de l'étang de Berre (1972) doivent leur réussite au génie végétal mis en place par les paysagistes : Pierre Pillet et Georges Demouchy. Leur choix s'est porté sur des végétaux spontanés ou rustiques adaptés et disposés en fonction de l'exposition aux embruns, mais n'a pas exclu l'artifice de l'exotisme sur des secteurs où l'emploi d'une espèce non autochtone peut mettre en valeur un espace (pied d'immeuble, arrière-cours, jardinet...). Ainsi, le pin pignon, Pinus pinea L., pour la Grande Motte, a été l'essence dominante de première ligne. Sa plantation en masse dans les alignements et les parcs au bord de la mer a permis à des espèces plus fragiles telles que les platanes, les peupliers blancs et les aulnes de résister en deuxième ligne. Ce gradient d'espèce a donc été pensé selon les caractéristiques et les inconvénients du site pour que, 50 ans plus tard, ces arbres forment une forêt urbaine artificielle. 
Figure 4. Vues du parc en direction de l'étang du Ponant
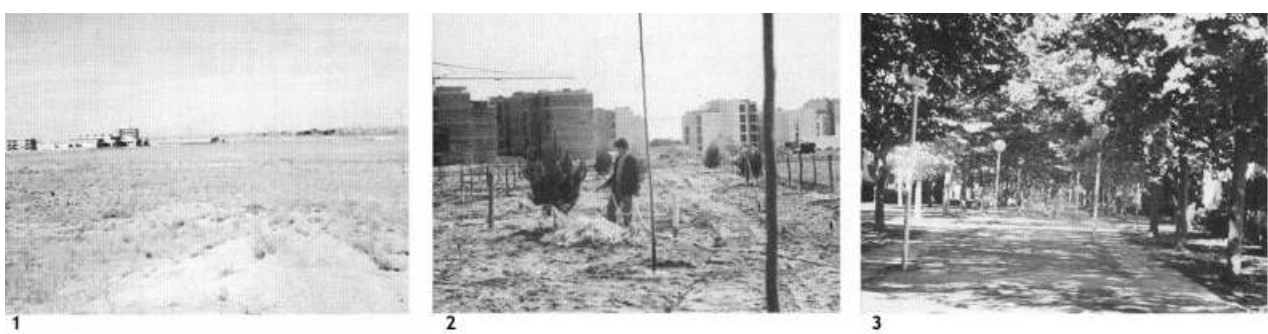

1. terrain remblayé, enjonqué, 1970 ; 2. plantations, 1974 ; 3. état des lieux, 1987

Source : Balladur et al., 1989.

Comme l'a démontré Anaïs Léger-Smith, l'émergence des considérations écologiques dans la société a fait naître chez les paysagistes une conscience ou un intérêt pour l'écologie. Mais peut-être faudrait-il aller plus loin en interrogeant ces professionnels sur leur histoire personnelle, leur enseignement dispensé dans les écoles de projet, notamment à l'École nationale supérieure de paysage de Versailles et leur intérêt (ou non) pour l'histoire de l'art des jardins afin de mieux comprendre ce qui influence aujourd'hui la relation entre la diversité végétale arborée et la pratique du projet de paysage.

\section{Conclusion}

L'approche chronologique que nous avons tenté d'illustrer au regard de l'arbre et de la diversité arborée dans les parcs, généralement privés avant le xix ${ }^{e}$ siècle, montre que le paysagiste a toujours eu une attention particulière à réserver un chapitre dans ses écrits sur l'arbre et les boisements. Malheureusement, depuis le premier quart du $\mathrm{xx}^{\mathrm{e}}$ siècle, nous ne trouvons plus de véritables traités d'art des jardins ou de traités relevant des jardins dans la ville, mais des ouvrages retraçant l'histoire des jardins en intégrant l'histoire des plantes (Wimmer, 2014), et l'histoire de la pratique du jardinage (Laird, 2014) comme éléments principaux.

Contre toute attente, l'esthétisation de la nature par le jardin au $\mathrm{XvII}^{\mathrm{e}}$ siècle semble montrer que les paysagistes ont su, à cette époque, installer les conditions nécessaires pour que les arbres expriment leur grandeur, surtout dans les espaces de second plan (bois, futaie, taillis...). Il existe aussi une disparité dans la structuration des phytocénoses forestières entre les jardins et les forêts des campagnes immédiates. Les unes, entretenues, sont destinées à l'agrément ou au plaisir de la chasse (cas d'un parc de chasse) tandis que les autres, exploitées, servent pour une multitude d'activités (agricoles, navales...), et présentent alors des formations végétales souvent dégradées. Des travaux récents sur les regarnis de Versailles et du Trianon (Olivesi, 2012) montrent cependant la difficulté et/ou la volonté de maintenir au cours du XVIII ${ }^{\mathrm{e}}$ siècle les jardins dans leur état d'origine mais aussi de nouveaux choix de gestion.

Le retour de la nature sylvestre, proche de l'idéal arcadien au XvIII siècle jusqu'au début du XIX ${ }^{e}$ siècle, a peut-être fait évoluer la diversité biologique dans les jardins par la distribution et la composition des masses boisées mais reste un art de l'imitation de la nature et surtout un archétype. En effet, le paysage forestier de la campagne environnante ne représente pas toujours la mise en scène pastorale des jardins car la forêt est là aussi souvent dégradée en friche ou en broussaille quand les bois jouxtent 
les terres vaines et les pâquis communaux (Husson, 1984). Pour affirmer ou infirmer l'évolution de la diversité biologique dans les jardins, il faudrait rassembler toutes les listes des végétaux présentes dans les ouvrages $d u x_{\text {XIII }}{ }^{\mathrm{e}}$ siècle mais aussi dans ceux $d u$ siècle passé $\left(\mathrm{xvII}^{\mathrm{e}}\right)$ et du siècle suivant $\left(\mathrm{xIX}^{\mathrm{e}}\right)$ en les croisant avec des outils de lexicométrie. À l'image de la base de données Hortus, de celle de l'Institut européen des jardins et des paysages ${ }^{5}$, etc., cela permettrait d'avoir une lecture qualitative et quantitative de la diversité arborée mais également de saisir plus objectivement, à travers ces trois derniers siècles, le choix des arbres, leur association et peut-être leur organisation dans l'espace en étudiant les différents plans de parcs et de jardins présents dans ces ouvrages.

En revanche, la surabondance d'espèces exotiques dans les parcs et jardins au XIX siècle, la perturbation des sols liés aux grands aménagements hygiénistes des villes et les entretiens abusifs des espaces plantés diminuent probablement les processus écosystémiques. De nombreux arbres ont d'ailleurs péri après l'arrêt des entretiens. À cette époque, le paysagiste n'est pas encore un savant pour les associations végétales, trop centré sur l'ingénierie à mettre en place pour répondre au dessein impérial, il reste dans le décor et l'artifice. Par conséquent, la modification de certains facteurs écologiques (abiotique, le sol; biotique, interaction entre les espèces exotiques et les espèces indigènes) a peut-être eu des incidences sur les relations intraspécifiques et interspécifiques entre les végétaux. L'étude des plans, la lecture plus fine de certains ouvrages de cette époque et les listes de végétaux pourront certainement donner des réponses plus précises.

Enfin, tout porte à croire que les paysagistes de l'après-guerre, nourris du traité d'Édouard André, des réalisations de Jean Claude Nicolas Forestier et de ses disciples, gardent de solides bases sur la manière de penser, de conduire l'arbre dans leurs réalisations, et cultivent un certain génie écologique malgré l'idéologie du Mouvement moderne.

51 Cependant, face à l'évolution rapide des concepts émergents au cours des 30 dernières années (nature en ville, écologie urbaine, gestion différenciée, biodiversité urbaine, etc.), face à l'évolution des discours et des formulations des commandes publiques, il est nécessaire de mettre en place des protocoles de recherche (choix de sites d'études, période d'étude, panel de paysagistes maîtres d'œuvre, etc.) et d'autres méthodes (analyse de discours, étude in situ de la diversité arborée dans les réalisations paysagères, etc.) pour continuer cette analyse. La ville de Marseille constituera alors le contexte de cette thèse avec un gradient potentiel de parcs ( 54 parcs $>1$ ha) des années 1850 à aujourd'hui. Ainsi, des tracés italianisants et classiques (Aillaud, 2011 ; Mihière, 1993) aux tracés pittoresques de Barillet-Deschamps (Limido, 2002), les parcs marseillais ont suivi l'évolution de l'art des jardins. Il est donc intéressant dans le cadre de cette thèse d'interroger, avec les connaissances actuelles en écologie, les paysagistes contemporains et la relation qu'il est possible d'apercevoir entre la diversité arborée et la reconstitution des milieux écologiques par la pratique du projet de paysage. Cela permettra en outre de questionner l'évolution des valeurs et des pratiques propres à la culture des professionnels du paysage et de répondre à cette interrogation : de quels facteurs dépend l'écologisation des pratiques paysagistes que nous analyserons à partir de plusieurs parcs choisis comme sites d'étude. praticiens à travers l'intégralité des traités de l'art des jardins de ces trois derniers 
siècles. Par-delà la différenciation du jardinier, de l'horticulteur et du paysagiste, cela permettra sûrement d'enrichir cette profession de savoirs oubliés, prêts à être réinvestis, de fournir des matériaux de recherche, pour croiser les valeurs et les pratiques du passé avec celles du présent, mais surtout, de nourrir une discipline, le paysagisme, en plein aggiornamento théorique.

\section{BIBLIOGRAPHIE}

Aggeri, G., « La nature sauvage et champêtre dans les villes : Origine et construction de la gestion différenciée des espaces verts publics et urbains. Le cas de la ville de Montpellier », thèse de doctorat en sciences de l'environnement, Sciences of the Universe, Engref, AgroParisTech, 2004.

Aggeri, G., Donadieu, P., « La nature sauvage dans les parcs urbains : du wild garden à la gestion différenciée ", Les Carnets du paysage, n 9-10, 2003, p. 171-187.

Aillaud, G. (dir.), Marseille un terroir et ses bastides, Marseille, Éditions du vieux Marseille, 2011.

Allorge, L., La Fabuleuse odyssée des plantes : les botanistes voyageurs, les jardins des plantes, les herbiers, Paris, Jean Claude Lattès, 2003.

Alphand, A., Les Promenades de Paris : histoire, description des embellissements, Paris, Rotschild, 1867-1973.

André, E., L'art des jardins. Traité général de la composition des parcs et jardins, Paris, Masson, 1879.

Arrif, T., Blanc, N., Clergeau, P., « Trame verte urbaine, un rapport Nature-Urbain entre géographie et écologie ", Cybergeo. Revue européenne de géographie, décembre 2011, URL : http:// journals.openedition.org/cybergeo/24862 ; DOI : https://doi.org/10.4000/cybergeo.24862

Arnould, P., Le Lay, F., Dodane, C., Meliani, I., « La nature en ville, l'improbable biodiversité », Géographie, Économie et Société, vol. 13, Paris, Lavoisier, 2011, p. 45-68.

Arnould, P., « Biodiversité, la confusion des chiffres et des territoires », Annales de géographie, Paris, Armand Colin, 2006, p. 528-549.

Balladur, J., Lanier, L., Pechairal, G., Pillet, P., « Une forêt urbaine entièrement artificielle : l'exemple de la Grande Motte », Revue forestière française, vol. 41, 1989, p. 171-187.

Baridon, M., Les Jardins : paysagistes, jardiniers, poètes, Paris, Robert Laffont, 1998.

Blanc, N., « 1925-1990 : l'écologie urbaine et le rapport ville-nature », Espace géographique, t. 27, nº 4, 1998, p. 289-299.

Blanchon, B., « Pratiques et compétences paysagistes dans les grands ensembles d'habitation, 1945-1975 », Strates, vol. 13, 2007, URL : http://strates.revues.org/5723.

Bossu, J., Candilis, G., Dalloz, P., De Looze, H., Lods, M., et al., « Le Corbusier témoignage », Nature et Architecture, $n^{\circ} 2,1966$.

Brunon, H., Chomarat-Ruiz, C., Donadieu, P., Torre, A., « Pour une « métascience du paysage », Projets de Paysages, $\mathrm{n}^{\circ}$ 2, juin 2009, URL : http://www.projetsdepaysage.fr/fr/ pour_une_metascience_du_paysage. 
Brunon, H., « Pratolino : art des jardins et imaginaire de la nature dans l'Italie de la seconde moitié du xvie siècle », thèse de doctorat, université Panthéon-Sorbonne-Paris 1, 2001.

Buridant, J., « la gestion des forêts de vénerie au XVII ${ }^{\mathrm{e}}$ siècle », Dix-Septième Siècle, $\mathrm{n}^{\circ}$ 226/1, 2005.

Chiesura, A., " The role of urban parks for the sustainable city ", Landscape and Urban Planning, $\mathrm{n}^{\circ}$ 68-1, 2004, p. 129-138.

Clergeau, P., «Préserver la nature dans la ville », Annales des Mines-responsabilité et environnement, vol. 52, Paris, Eska, 2008, p. 52-59.

Deleage, J.-P., « Aux origines de la science écologique : à propos de quelques ouvrages récents », Revue d'histoire des sciences, $\mathrm{n}^{\circ}$ 45-4, 1992, p. 477-490.

Dezallier d'Argenville, A. J., La théorie et la pratique du jardinage où l'on traite à fond des beaux jardins appelés communément jardins de propreté, Paris, Mariette, J., 1709.

Donadieu, P., Sciences du paysage entre théories et pratiques, Paris, Lavoisier, Coll. « TEC \& DOC », 2012.

Donadieu, P., « Où vont les architectes paysagistes ? Du paysagisme jardiniste au paysagisme de médiation ", XII Conferenza Nazionale Società degli Urbanisti, il progetto dell' urbanistica per paesaggio, colloque du Bari, 19 février 2009a, URL : www.planum.net.

Donadieu, P., Les Paysagistes, Arles et Versailles, Actes Sud, École nationale supérieure du paysage, $2009 \mathrm{~b}$.

Donadieu, P., « Comment évolue le rôle du végétal dans la cité ? », colloque « La plante dans la ville », Angers, INRA édition, 1996, p. 21-27.

Dubost, F., Vert patrimoine : la constitution d'un nouveau domaine patrimonial, Paris, Édition de la Maison des sciences de l'homme, 1994.

Duhamel de Monceau, H.-L., Traité des arbres et arbustes qui se cultivent en France en pleine terre, t. 2, Paris, H.-L. Guérin et L.-F. Delatour, 1755.

Forestier, J. C. N., Grandes villes et Systèmes de parcs, Paris, Hachette, 1908.

Girardin, R.-L. de, De la composition des paysages ou les moyens d'embellir la nature autour des habitations, en joignant l'agréable à l'utile (1777), Ceyzérieu, Champ Vallon, 2014.

Hortus, base de données du Centre de recherche du château de Versailles, http:// www.chateauversailles-recherche-ressources.fr/jlbweb/jlbWeb?html=accueilhortus. Husson, J.-P., « Les lisières forestières au XVIII ${ }^{\mathrm{e}}$ siècle. L'exemple lorrain », Revue forestière française, vol. 36, n 5, 1984, p. 404-425.

Hautecoeur, L., Les Jardins des dieux et des hommes, Paris, Hachette, 1959.

Le Guyader, H., « La biodiversité : un concept flou ou une réalité scientifique ? ", Courrier de l'environnement de l'Inra, $\mathrm{n}^{\circ}$ 55, 2008, p. 7-26.

Laird, M., A natural of English gardening 1650-1800, Yale, Yale University Press, 2014.

Léger-Smith, F.-A., «Évolution des pratiques paysagistes face aux enjeux écologiques de la conception urbaine ", thèse de doctorat en architecture, space management, université d'Angers, 2014.

Limido, L., L'Art des jardins sous le Second Empire. Jean-Pierre Barillet-Deschamps, Seyssel, Champs vallon, 2002. 
Medhi, L., Weber, C., Pietro, F.-D., Selmi, V., «Évolution de la place du végétal dans la ville, de l'espace vert à la trame verte ", Vertigo. La revue électronique des sciences de l'environnement, vol. 12, $\mathrm{n}^{\circ}$ 2, 2012, URL : http://vertigo.revues.org/12670.

Mihière, G., Les Bastides marseillaises, de la villégiature en Provence, Marseille, Éditions Jeanne Laffitte, 1993.

Mollie, C., Des arbres dans la ville : l'urbanisme végétal, Arles, Actes Sud, 2009.

Mosser, M., Teyssot, G., Histoire des jardins de la renaissance à nos jours, Paris, Flammarion, 2002.

Oesterle, G., « Révolution des jardins et culture du souvenir », Revue germanique internationale, $\mathrm{n}^{\circ}$ 7, 1997.

Olivesi, F., « Les regarnis de Versailles et de Trianon à travers vingt commandes aux pépinières du roi (1753-1772) », Bulletin du Centre de recherche du château de Versailles, novembre 2012, URL : http://journals.openedition.org/crcv/11876 ; DOI : https://doi.org/10.4000/crcv.11876

Pousin, F., «Qu'attendre de la production des paysages contemporains ? », Critique d'art, $\mathrm{n}^{\circ} 26$, automne 2005, URL : http://critiquedart.revues.org/1126.

Quellier, F., «L'automne horticole du Moyen Âge, permanences médiévales dans les traités de jardinage de la première modernité (1486-1652) ", Archéologie du midi médiéval, vol. 23-24, 2005, p. 109-119.

Quenet, G., Versailles, une histoire naturelle, Paris, La découverte, 2015.

Raymond, R., Simon, L., « Biodiversité : les services écosystémiques de la nature en ville », Revue forestière française, $\mathrm{n}^{\circ}$ 3, 2012, p. 339-350.

Sahut, F., « L'horticulteur du XIX siècle », Revue horticole, Paris, la Maison Rustique, 1859.

Searns, R.-M., « The evolution of greenways as an adaptative urban landscape form ", Landscape and urban planning, vol. 33, 1995, p. 65-80.

Serres, O. de, Le Théâtre de d'agriculture et mesnage des champs, Paris, Métayer, 1600.

Tollis, C., «Pour le meilleur et pour le pire! Les arbres en ville peuvent-ils faire patrimoine? Analyse des spatialités concurrentes arbres-riverains à Grenoble », Vertigo. La revue électronique des sciences de l'environnement, hors série $\mathrm{n}^{\circ} 16$, mai 2013, URL : http://journals.openedition.org/ vertigo/13736; DOI : https://doi.org/10.4000/vertigo.13736

Vergnaud, N., Disponzo, J., L'Art de créer les jardins, Saint Nazaire, Les éditions Petit Génie, 2015.

Werquin, A.-C., Demangeon, A., «L'entrelacs du végétal et de l'urbanisation », Les Annales de la recherche urbaine. Natures en ville, $\mathrm{n}^{\circ} 74,1997$, p. 40-47.

Wimmer, C.-W., Lustwald, Beet und Rosenhügel : Geschichte der Pflanzenverwendung in der Gartenkunst, Weimar, Verlag und Datenbank für Geisteswissenschaft, 2014.

Wimmer, C.-W., Geschichte der Gartentheorie, Darmstadt, Wissenschaftliche Buchgesellschaft, 1989.

\section{NOTES}

1. Rattachée à la discipline de la botanique, l'organographie végétale étudie la description et l'étude des organes végétaux.

2. Au $\mathrm{I}^{\mathrm{er}}$ siècle av. J.-C, Dioscoride (médecin et botaniste grec) véhicule l'idée que tout l'art de la médecine consiste à utiliser des médicaments ressemblant aux organes malades. 
3. Cette base de données du centre de recherche du château de Versailles rassemble des sources traitant du végétal dans les grands jardins européens à l'époque moderne (http:// chateauversailles-recherche.fr).

4. «L'instrument imaginé par M. Mazure est très suffisant pour donner les proportions du sable, du calcaire et de l'argile contenus dans la terre dont on désire connaître la composition » (André, 1879, p. 598).

5. L'Institut européen des jardins et des paysage rassemble des archives, des inventaires publics et privés relatifs à l'art des jardins en Europe (http://europeangardens.eu).

\section{RÉSUMÉS}

Cet article vise à interroger la relation entre la diversité arborée et la pratique du projet de paysage à travers la littérature existante d'histoire des jardins publiée au cours des trois derniers siècles. La lecture de ces documents a permis d'identifier quatre grandes "ères » qui ont non seulement fait évoluer des manières de penser et de dessiner les projets, mais aussi d'intégrer et de conduire l'arbre dans les aménagements de grands parcs et domaines privés du XVII ${ }^{\mathrm{e}}$ siècle à ceux d'aujourd'hui, rendus publics et urbains. Ainsi, du XVII ${ }^{\mathrm{e}}$ au $\mathrm{Xx}^{\mathrm{e}}$ siècle, de l'art des jardins classique à l'urbanisme fonctionnaliste, nous verrons comment il est déjà possible de dégager des paramètres d'organisation, de structure et de fonctionnement qui témoignent avant l'heure d'une dimension de la diversité arborée. Cela a pour objectif de replacer dans l'histoire cet élément de nature présent dans les villes comme un vecteur et/ou un support de biodiversité.

This article focuses on the relation between arborescent plant diversity and the landscape project in the literature of the history of gardens published during the last three centuries. The study if these documents has made it possible to identify four major "eras" which not only contributed to changing the way of thinking about and designing projects, but also integrated and used the arborescent plant as a defining component in the development of major parks and private domains from the 17th century up until today, and which have now become public and urban spaces. Thus, from the 17th to the 20th century, spanning from the art of classic gardens to the trend of functional urbanism, the article shows how it is possible to identify the parameters relating to forms of organisation, structure and practise which point to the early presence of tree diversity. The objective is to place within the historical framework this natural element present in cities as a vector of and/or a support for biodiversity.

\section{INDEX}

Mots-clés : conception, biodiversité, arbre, parcs/jardins, ville, projet

Keywords : design, biodiversity, tree, parks/gardens, city, project 


\section{AUTEURS}

\section{BRICE DACHEUX-AUZIÈRE}

Paysagiste DPLG, Brice Dacheux-Auzière est doctorant à l'École nationale supérieure de Paysage (ENSP - site de Marseille), Laboratoire de recherche en paysage (Larep).

brice-dacheux[at]wanadoo[dot]fr

\section{AUDREY MARCO}

Audrey Marco est maître de conférences en spatialisation de projet et démarche écologie à l'École Nationale Supérieure de Paysage (ENSP - site de Marseille), Laboratoire de recherche en paysage (Larep).

a.marco[at]ecole-paysage[dot]fr

\section{YVES PETIT-BERGHEM}

Yves Petit-Berghem est professeur d'écologie à l'École nationale supérieure de paysage (ENSP site de Versailles), Laboratoire de recherche en paysage (Larep).

y.petitberghem[at]ecole-paysage[dot]fr 